Comunicação Breve

Brief Communication

Alexandre Lucas de Araújo Barbosa ${ }^{1}$ (c) Cíntia Alves Salgado Azoni' ${ }^{1}$ (c)

Descritores

Estudos de Validação Programas de Rastreamento

Linguagem Infantil Protocolos

Testes de Vocabulário Vocabulário

Keywords

Validation Studies

Mass Screening Infant Language

Protocols

Vocabulary Tests

Vocabulary

Endereço para correspondência: Alexandre Lucas de Araújo Barbosa Departamento de Fonoaudiologia, Centro de Ciências da Saúde, Universidade Federal do Rio Grande do Norte - UFRN

Rua Poeta José Revoredo Neto, 105, Nova Parnamirim, Parnamirim (RN), Brasil, CEP: 59151-380.

E-mail: ilucasbarbosa@icloud.com

Recebido em: Janeiro 09, 2020

\section{Validade baseada nos processos de resposta do trilhar - instrumento de triagem}

\author{
do vocabulário infantil
}

\author{
Validity evidence based on response \\ processes of the TRILHAR - screening tool for \\ infant vocabulary
}

\begin{abstract}
RESUMO
Objetivo: Verificar as evidências de validade baseadas nos processos de resposta de um instrumento de triagem do vocabulário. Método: Trata-se de um estudo descritivo, transversal e quantitativo, aplicado em uma amostra de 133 crianças entre 3 e 7 anos de idade, subdivididos em cinco grupos de acordo a faixa etária. $\mathrm{O}$ instrumento sobre o qual se trata a pesquisa é o TRILHAR, que visa a triagem do vocabulário receptivo e expressivo, composto por dez fichas de atividades para cada idade-alvo. Os dados coletados focaram no tempo de aplicação em minutos e segundos, compreensão das instruções do teste e comportamento do escolar durante a aplicação. Os dados foram analisados descritivamente em relação a média, desvio-padrão e porcentagem por meio do software IBM SPSS Statistics. Resultados: O tempo de aplicação foi curto e diminuiu de acordo com a progressão da idade. O tempo médio de aplicação foi de $05 \mathrm{~m} 19 \mathrm{~s}$ para o $\mathrm{G} 1,04 \mathrm{~m} 29 \mathrm{~s}$ para o $\mathrm{G} 2,04 \mathrm{~m} 11 \mathrm{~s}$ para o G3, 03m40s para o G4 e $02 \mathrm{~m} 13$ s para o G5. Apenas um sujeito (4,8\%) do G2 necessitou de repetição das instruções no vocabulário receptivo e dois $(6,3 \%)$ do G4 no vocabulário expressivo. Foi verificada pequena ocorrência de comportamentos como desinteresse, desatenção e agitação. Conclusão: Foi verificado que o tempo de aplicação do instrumento necessita de um curto período de tempo, sendo mais dispendiosa no grupo de crianças com três anos de idade. A baixa necessidade de repetição das instruções indica que o instrumento apresenta fácil compreensão por parte da criança.
\end{abstract}

\begin{abstract}
Purpose: To verify the validity evidence based on response processes of a vocabulary-screening tool. Methods: This is a descriptive, cross-sectional and quantitative study, applied in a sample of 133 children between 3 and 7 years of age, divided into five groups, according to their age range. This research evaluates the instrument TRILHAR, which is a screening of receptive and expressive vocabulary, composed by ten activities for each age range. The collected data were the application time in minutes and seconds, screening instructions comprehension and behavior during the screening. The data were analyzed descriptively as mean, standard deviation and percentage using the IBM SPSS Statistics. Results: The application time was short, with less time for the greater ages. The mean time of application was $05 \mathrm{~m} 19 \mathrm{~s}$ for G1, 04m29s for G2, $04 \mathrm{~m} 11 \mathrm{~s}$ for G3, 03m $40 \mathrm{~s}$ for G4 and $02 \mathrm{~m} 13 \mathrm{~s}$ for G5. Only one child (4.8\%) from G2 required repetition of the instructions for the receptive vocabulary, and two children $(6.3 \%)$ from G4 for the expressive vocabulary. We observed little occurrence of behaviors like disinterest, inattention and agitation. Conclusion: The application of the instrument required a short period, with a longer time for the group of 3-years children. The little necessity of repetition of the instructions indicates that children can easily comprehend the instrument.
\end{abstract}

Trabalho realizado no Departamento de Fonoaudiologia, em parceria com o Departamento de Engenharia Biomédica, Universidade Federal do Rio Grande do Norte - UFRN - Natal (RN), Brasil.

${ }^{1}$ Departamento de Fonoaudiologia, Centro de Ciências da Saúde, Universidade Federal do Rio Grande do Norte - UFRN - Natal (RN), Brasil.

Fonte de financiamento: Bolsa de mestrado concedida pela Coordenação de Aperfeiçoamento de Pessoal de Nível Superior (Capes), processo $\mathrm{n}^{\circ} 1728008$

Conflito de interesses: nada a declarar. 


\section{INTRODUÇÃO}

Um teste de triagem é um procedimento aplicado em indivíduos saudáveis e não-saudáveis, visando identificar aqueles que apresentam sinais de risco quanto ao desenvolvimento de determinada condição ou doença, para que a intervenção precoce traga melhores resultados ${ }^{(1)}$. Para que seja efetivo, um teste de triagem deve ser validado, de simples compreensão e aplicação, possuir reprodutibilidade e acurácia, além de existirem oportunidades de tratamento para os indivíduos-alvo ${ }^{(2-4)}$.

A validade de processos de resposta verifica os processos psicológicos, cognitivos e sociais envolvidos na aplicação do instrumento, tais como dificuldades operacionais, tempo de aplicação, reações não verbais e compreensão do indivíduo testado acerca das instruções e questões ${ }^{(5)}$. Uma das formas mais utilizadas de obter a validade de resposta é por meio de entrevista cognitiva ${ }^{(6)}$.

No cenário da Fonoaudiologia do Brasil, as pesquisas referentes ao processo de validação de instrumentos são escassas ${ }^{(7)}$. Em relação à triagem do vocabulário infantil, este cenário se reafirma, indicando a necessidade de pesquisas nesta área ${ }^{(8)}$, pois a identificação e intervenção precoce traz resultados longitudinalmente positivos no desenvolvimento da linguagem do indivíduo ${ }^{(9)}$. Crianças com atrasos no desenvolvimento da linguagem que não sejam submetidas a intervenção, tendem a manter desempenho aquém de seus pares típicos ao longo da vida ${ }^{(10)}$.

Tendo em vista os dados apresentados acima e a escassez de instrumentos de triagem de vocabulário no cenário de pesquisa e clínica brasileira, o objetivo deste estudo foi verificar as evidências de validade baseadas nos processos de resposta de um instrumento de triagem do vocabulário para crianças entre 3 e 7 anos de idade.

\section{MÉTODO}

Esta é uma pesquisa do tipo descritiva, transversal e quantitativa, aprovada pelo Comitê de Ética em Pesquisa (CEP), parecer número 2.548.341. Todos os participantes assinaram o Termo de Consentimento Livre e Esclarecido (TCLE) e Termo de Assentimento Livre e Esclarecido (TALE), se necessário para crianças acima dos 7 anos de idade.

O tamanho da amostra baseou-se na literatura nacional, de que para a etapa de validação de acordo com os processos de resposta, deve ser composta por ao menos dez indivíduos de cada grupo ${ }^{(5)}$. A amostra, de conveniência, foi composta por 133 crianças entre 3 e 7 anos, matriculadas no ensino regular público, sem queixas de alterações no desenvolvimento da linguagem ou diagnósticos de síndromes e deficiências, de acordo com o relato das professoras. Foram divididos nos seguintes grupos, considerando-se a faixa etária:

- Grupo 1 - 38 indivíduos com três anos de idade;

- Grupo 2 - 21 indivíduos com quatro anos de idade;

- Grupo 3 - 30 indivíduos com cinco anos de idade;

- Grupo 4 - 32 indivíduos com seis anos de idade;

- Grupo 5 - 22 indivíduos com sete anos de idade.
Este estudo trata-se de uma etapa da validação do instrumento de triagem do vocabulário receptivo e expressivo - TRILHAR ${ }^{(8)}$, indicado para crianças entre três e sete anos de idade. Seu objetivo é identificar precocemente pré-escolares e escolares com sinais de risco para alterações relacionadas ao nível semântico da linguagem, especialmente no âmbito educacional, visando a intervenção precoce e prevenção de possíveis dificuldades consequentes à restrição do vocabulário.

O TRILHAR é composto por dez fichas de vocabulário receptivo, nas quais a criança ouve uma palavra e aponta para a figura correspondente dentre 4 oferecidas simultaneamente e; dez fichas de vocabulário expressivo, na qual deve ser feita a nomeação de uma única figura exibida. As figuras da triagem são específicas e diferenciadas para cada faixa etária englobada pelo instrumento, selecionadas e distribuídas de acordo com critérios, os quais podem ser visualizados no artigo referenciado que relata a construção do teste de triagem ${ }^{(8)}$. É composto por palavras das seguintes categorias semânticas: roupas, animais, alimentos, móveis, utensílios, meios de transporte, brinquedos, instrumentos, profissões, lugares, partes do corpo, adjetivos e verbos. A pontuação máxima é de vinte pontos, sendo dez da atividade receptiva e dez da expressiva.

Para a aplicação foram contatadas duas escolas públicas do ensino infantil e fundamental para a obtenção da anuência. Subsequentemente, os pais foram convidados para participação em uma reunião, com explicações acerca da pesquisa e do funcionamento do instrumento. Ao final, o TCLE e TALE foram assinados. Todas as triagens foram aplicadas individualmente, em sala com condições acústicas e de iluminação adequadas, no horário combinado com as professoras de cada turma, utilizando o material impresso do instrumento, com apoio do fantoche para motivação da criança. A aplicação foi iniciada pelo vocabulário receptivo, seguido pelo vocabulário expressivo.

A instrução utilizada para a triagem do vocabulário receptivo foi a seguinte: "Vou te mostrar quatro desenhos e vou te pedir para apontar uma delas". Já para o vocabulário expressivo: "Vou mostrar uma figura e quero que você fale o nome dela". Para verificar a compreensão das instruções, foi utilizada a estratégia de paráfrase ${ }^{(5)}$, que consiste na repetição da ordem pelo indivíduo. Após a explicação da atividade, foi perguntado para a criança se ela compreendeu e, em seguida, pedido para que repetisse o que havia sido falado.

Para complementar a análise de compreensão das instruções, foram registrados os seguintes comportamentos que poderiam indicar dificuldades operacionais ${ }^{(5)}$ para a realização da triagem: desinteresse, expressões faciais de estranhamento, agitação, negativa de resposta, irritação, levantou da cadeira e choro.

A contagem do tempo de aplicação da triagem foi realizada por meio da utilização de um cronômetro nos celulares dos pesquisadores, sendo registrada no formato minutos e segundos. O início da contagem ocorreu a partir da explicação das instruções e foi finalizado ao final da triagem, englobando os vocabulários receptivo e expressivo.

Todos os dados foram analisados descritivamente, com uso do IBM SPSS Statistics, versão 23. 


\section{RESULTADOS}

Em todos os grupos, houve alta taxa de compreensão das instruções e baixa porcentagem necessidade de repetição para compreensão (Tabela 1). As principais observações comportamentais foram as expressões faciais de estranhamento e agitação (Tabela 2).

Por fim, o tempo de aplicação dos vocabulários receptivo e expressivo diminuiu de acordo com a progressão da faixa etária. O G1 apresentou tempo de aplicação de $05 \mathrm{~m} 29 \mathrm{~s}(\mathrm{dp}=01 \mathrm{~m} 41 \mathrm{~s})$, seguido pelo $\mathrm{G} 2$ com $04 \mathrm{~m} 29 \mathrm{~s}(\mathrm{dp}=01 \mathrm{~m} 19 \mathrm{~s})$. O G3 completou o teste em $04 \mathrm{~m} 11 \mathrm{~s}(\mathrm{dp}=01 \mathrm{~m} 12 \mathrm{~s}), \mathrm{o}$ G4 em 03m40s $(\mathrm{dp}=01 \mathrm{~m} 12 \mathrm{~s})$ e $\mathrm{G} 502 \mathrm{~m} 13 \mathrm{~s}(\mathrm{dp}=47 \mathrm{~s})$.

\section{DISCUSSÃO}

De acordo com os resultados deste estudo, verificou-se que o TRILHAR possui validade de processos de resposta satisfatória. As características comportamentais observadas indicam que o instrumento foi capaz de reter a atenção da criança, sendo de simples aplicação. Assim, estes achados são importantes, pois condiz com o procedimento de triagem, que deve ser simples e rápido ${ }^{(2-4)}$, como observado na aplicação do instrumento na amostra. A capacidade de reter a atenção da criança é de extrema importância, visto que diminui as chances de resultados desfavoráveis devido a fatores como desatenção e falta de interesse. Isto deve-se ao fato de as figuras do instrumento serem construídas visando aplicação lúdica, bem como a utilização do fantoche torna o ambiente mais interessante para o indivíduo, favorecendo sua participação ativa no processo de triagem.

Quanto as instruções do instrumento, apenas uma criança do G2 e duas crianças do G4 necessitaram de maiores informações e não conseguiram repetir as indicações na estratégia de paráfrase. Esta é uma característica positiva do teste, visto que instruções de qualidade devem ser claras e precisas para a população a qual o teste se direciona ${ }^{(11)}$. A possibilidade de desempenhos baixos devido a instruções falhas é alta, justificando a atenção criteriosa a este aspecto.

O tempo médio de aplicação diminuiu de acordo com o aumento da faixa etária, o que provavelmente pode ser explicado pelas características do desenvolvimento típico do vocabulário, ou seja, quanto maior a idade, o léxico da criança é composto por mais palavras ${ }^{(12)}$. Este fator facilitou não somente a compreensão das instruções, como também a velocidade de resposta da criança, diminuindo assim o tempo de aplicação. Outra possível explicação é de que as crianças mais velhas apresentam melhor desenvolvimento atencional ${ }^{(13)}$, diminuindo assim a necessidade de tempo adicional para resposta do teste. O TRILHAR foi construído visando curto tempo de aplicação e, por isso, é composto por 10 itens de vocabulário receptivo e 10 de vocabulário expressivo para cada faixa etária.

Uma limitação da pesquisa é a não exploração dos dados mais qualitativos referentes à validação baseadas no processo de resposta. Estudos futuros devem ser conduzidos para verificar se o tipo de instrução oferecida à criança influencia na qualidade de suas respostas, mais especificamente em sua pontuação no teste. Outra limitação refere-se a amostra do estudo, visto que foi composta por crianças estudantes de uma escola pública de referência. Também é importante comparar o desempenho de crianças em situação de vulnerabilidade socioeconômica, pois estas podem ter maiores dificuldades em vocabulário ${ }^{(14)}$, o que pode influenciar diretamente na compreensão das instruções e desempenho nos resultados finais do instrumento, visto que a amostra deste artigo foi composta por crianças estudantes de uma escola pública de referência na região. Considera-se também necessária a aplicação em estudantes de escolas da rede privada, pois estes podem apresentar melhores resultados em provas de vocabulário ${ }^{(15)}$.

Tabela 1. Porcentagem de compreensão das ordens repetições necessárias das instruções da triagem

\begin{tabular}{lcccccc}
\hline & & G1 & G2 & G3 & G4 & G5 \\
\hline Vocabulário receptivo & Compreensão & $100 \%(n=28)$ & $95,2 \%(n=20)$ & $100 \%(n=30)$ & $100 \%(n=32)$ & $100 \%(n=22)$ \\
& Repetições & $0 \%(n=0)$ & $4,8 \%(n=1)$ & $0 \%(n=0)$ & $3,1 \%(n=1)$ & $0 \%(n=0)$ \\
Vocabulário expressivo & Compreensão & $100 \%(n=28)$ & $95,2 \%(n=20)$ & $100 \%(n=30)$ & $93,8 \%(n=30)$ & $100 \%(n=22)$ \\
& Repetições & $0 \%(n=0)$ & $0 \%(n=0)$ & $0 \%(n=0)$ & $6,3 \%(n=2)$ & $0 \%(n=0)$ \\
\hline
\end{tabular}

Legenda: G1 = grupo 1; G2 = grupo 2; G3 = grupo 3; G4 = grupo 4; G5 = grupo 5

Tabela 2. Ocorrência (n) das características comportamentais observadas

\begin{tabular}{|c|c|c|c|c|c|}
\hline & G1 (n) & G2 (n) & G3 (n) & G4 (n) & G5 (n) \\
\hline Desinteresse & 0 & 3 & 0 & 1 & 0 \\
\hline Expressões faciais de estranhamento & 0 & 1 & 3 & 3 & 0 \\
\hline Agitação & 4 & 0 & 4 & 1 & 0 \\
\hline Negou resposta & 1 & 1 & 1 & 0 & 0 \\
\hline Irritação & 0 & 0 & 0 & 0 & 0 \\
\hline Levantou da cadeira & 0 & 0 & 1 & 0 & 0 \\
\hline Chorou & 0 & 0 & 0 & 0 & 0 \\
\hline
\end{tabular}

Legenda: G1 = grupo 1; G2 = grupo 2; G3 = grupo 3; G4 = grupo 4; G5 = grupo 5 


\section{CONCLUSÃO}

A partir da análise dos processos de resposta, verificou-se que o TRILHAR é um instrumento de fácil compreensão pela criança, visto que apenas um indivíduo do G2 e dois indivíduos do G4 apresentaram dificuldades neste aspecto. $\mathrm{O}$ teste apresenta baixo tempo de aplicação, características compatíveis com a definição do procedimento de triagem: média de $05 \mathrm{~m} 19 \mathrm{~s}$ para o G1, 04m29s para o G2, $04 \mathrm{~m} 11$ s para o $\mathrm{G} 3,03 \mathrm{~m} 40$ s para o G4 e $02 \mathrm{~m} 13 \mathrm{~s}$ para o G5. São necessárias pesquisas futuras para complementar o instrumento com outros dados de validade, como a consistência interna, confiabilidade e acurácia.

\section{AGRADECIMENTOS}

À Coordenação de Aperfeiçoamento de Pessoal de Nível Superior (Capes) pelo apoio para a realização desta pesquisa.

\section{REFERÊNCIAS}

1. Maxim L, Niebo R, Utell M. Screening tests: a review with examples. Inhal Toxicol. 2014;26(13):811-28. http://dx.doi.org/10.3109/08958378 .2014.955932. PMid:25264934.

2. Herman C, Gill H, Eng J, Farjado L. Fundamentals of clinical research for radiologists. AJR Am J Roentgenol. 2002;179(4):825-31. http://dx.doi. org/10.2214/ajr.179.4.1790825. PMid:12239019.

3. Bliss L, Allen D. Screening kit of language development: a preschool language screening instrument. J Commun Disord. 1984;17(2):133-41. http://dx.doi.org/10.1016/0021-9924(84)90019-4. PMid:6725626.

4. Wilson J, Jungner G. Principles and practice of screening for disease. J R Coll Gen Pract. 1968;16(4):281-393. PMid:4234760.

5. Pernambuco L, Espelt A, Magalhães HV Jr, Lima KC. Recommendations for elaboration, transcultural adaptation and valitation process of tests in speech, hearing and language pathology. CoDAS. 2017;29(3):e20160217. PMid:28614460.
6. Padilla J, Benítez I. Validity evidence based on response processes. Psicothema. 2014;26(1):136-44. PMid:24444741.

7. Gurgel LG, Kaiser V, Reppold TZ. A busca de evidências de validade no desenvolvimento de instrumentos em Fonoaudiologia: revisão sistemática. Audiol Commun Res. 2015;20(4):371-83. http://dx.doi.org/10.1590/23176431-2015-1600.

8. Barbosa A, Soares H, Azoni C. Construção de um instrumento de triagem do vocabulário para crianças entre 3 e 7 anos. Audiol Commun Res. 2019;24:e2131. http://dx.doi.org/10.1590/2317-6431-2019-2131.

9. Fricke S, Bowyer-Crane C, Haley AJ, Hulme C, Snowling MJ. Efficacy of language intervention in the early years. J Child Psychol Psychiatry. 2013;54(3):280-90. http://dx.doi.org/10.1111/jcpp.12010. PMid:23176547.

10. Rescorla L. Age 17 language and reading outcomes in late-talking toddlers: support for a dimensional perspective on language delay. J Speech Lang Hear Res. 2009;52(1):16-30. http://dx.doi.org/10.1044/1092-4388(2008/070171). PMid: 18723598 .

11. Noronha APP. Análise de testes de personalidade: qualidade do material, das instruções, da documentação e dos itens qualidade de testes de personalidade. Rev Estudos de Psicologia. 2002;19(3):55-65. http://dx.doi.org/10.1590/ S0103-166X2002000300006.

12. Cáceres-Assenço A, Ferreira S, Santos A, Befi-Lopes D. Application of a brazilian text of expressive vocabulary in European Portuguese children. CoDAS. 2018;30(2):e20170113. PMid:29791612.

13. Luria A. Curso de psicologia geral. 1. ed. Rio de Janeiro: Civilização Brasileira; 1979.

14. Jacobsen G, Moraes A, Wagner F, Trentini C. Qual é a participação de fatores socioeconômicos na inteligência de crianças. Rev Neuropsicol Latinoam. 2013;5(4):32-8.

15. Brancalioni A, Zauza A, Karlinski C, Quitaiski L, Thomaz M. Desempenho do vocabulário expressivo de pré-escolares de 4 a 5 anos da rede pública e particular de ensino. Audiol Commun Res. 2018;23(0):e1836. http:// dx.doi.org/10.1590/2317-6431-2016-1836.

\section{Contribuição dos autores}

ALAB e CASA participaram da concepção e delineamento do estudo, coleta, análise e interpretação dos resultados; ALAB e CASA participaram da redação e revisão do artigo de forma intelectualmente importante. 